\title{
Enhanced Smart Edge Broker using Fog Computing for Smart Homes
}

\author{
Junguk Ahn ${ }^{1}$, Byung Mun Lee ${ }^{2 *}$ \\ ${ }^{1}$ Gachon University, Dept. of IT Convergence, \\ ${ }^{2}$ Gachon University, Dept. of Computer Engineering, \\ ${ }^{1}$ woodencatty@gmail.com, ${ }^{2}$ bmlee@gachon.ac.kr
}

\begin{abstract}
Smart homes with IoT technologies provide convenience in our daily lives. If it is linked to smart city infrastructure through the expansion of service range, it can provide higher quality services. However, if the service range becomes wider, it may increase latency, which may in turn cause an overload. These problems are attributable to the increasing number of sensors that need to be monitored and the expansion of the service area. These problems can be solved by intelligently distributing the data measured in each area. Therefore, in this paper, we propose a Smart Edge Broker to intelligently transmit data traffic generated by a smart city in a wide area to prevent the traffic from being transmitted or bypassed to an area where traffic is not necessary. It is also suitable to operate it as a fog computing model by placing it at the edge of a smart city network.
\end{abstract}

Keywords: IoT; Smart City; Smart Home; Fog Computing

\section{Introduction}

To provide convenience in our daily lives, it is possible to configure a smart home using various IoT technologies[1][2]. For example, if we attach health sensors such as an ECG recorder and blood glucose meters to our body and link them to a smart home system, it is possible to remotely monitor our health[3]. Alternatively, by linking a sleep care device to a smart home system, it is possible to measure sleep and create an environment for improving sleep[4]. If it is linked to a smart city platform, it can provide a variety of services[5][6]. For example, it can monitor the activities of elderly people and people with dementia at home and provide more complex medical support services through the urban infrastructure in the event of an emergency[7]. However, as the coverage of the network becomes broader, the number of sensor devices increases, which may cause several problems [8][9].

First, there is a latency problem. This means that it takes a long time to transmit because the transmission distance of sensor data increases. If the distance between smart home sensors that generate data and a service that consumes the data increases, there will be latency in transmission. This will impair the immediateness of sensor data, thereby lowering the quality of the service. Second, there is an overload problem. When measuring by increasing the number of sensors and widening the range, the amount of the data that needs to be processed increases, which may lead to bottlenecks due to an excessive concentration of traffic in a specific data center. This will delay data processing and service provision. Third, there is a service scalability

Article history:

Received (February 10, 2019), Review Result (March 9, 2019), Accepted (May 12, 2019) 
problem. When new services are added to a smart home and smart city, all the related services need to be changed, increasing the cost as a result. These problems need to be solved when a smart city is configured. To that end, an intelligent distributed processing technique between sensors and services is needed.

In this paper, we propose a Smart Edge Broker(SEB) as an intelligent distributed processing technique. The Smart Edge Broker, located at the edge between sensors, services, and data centers distributed in the city, intelligently routes and brokers data transmission. Through this process, the data can be directly transmitted between sensors, thereby reducing the distance and latency. As it also processes the data transmission between sensors and services instead of data centers, it naturally solves the overload problem. In addition, if the Smart Edge Broker can filter only the necessary data, scalability can be improved.

In Chapter 2, we examine smart homes and smart cities to identify likely problems. In Chapter 3, we define a Smart Edge Broker that can solve the problems of a smart city. In Chapter 4, we conclude based on the results of experiments and evaluations.

\section{Related Studies}

A smart home is an automation system that not only remotely controls home appliances in a house by connecting them to a network, but also detects and actively controls situations using sensors[10]. Figure 1 shows an example of a sleep care service. The sleep environment and biometric sleep information measured from sensors in each house are transmitted to an IoT sleep care service provided by the cloud[11]. Each Smart Home A, B, C, and D has a sound sensor and a temperature sensor, and a service gateway that collects the measured data and transmits it to IoT sleep care service. This service collects and analyzes the data from each smart home and provides sleep care services to users.

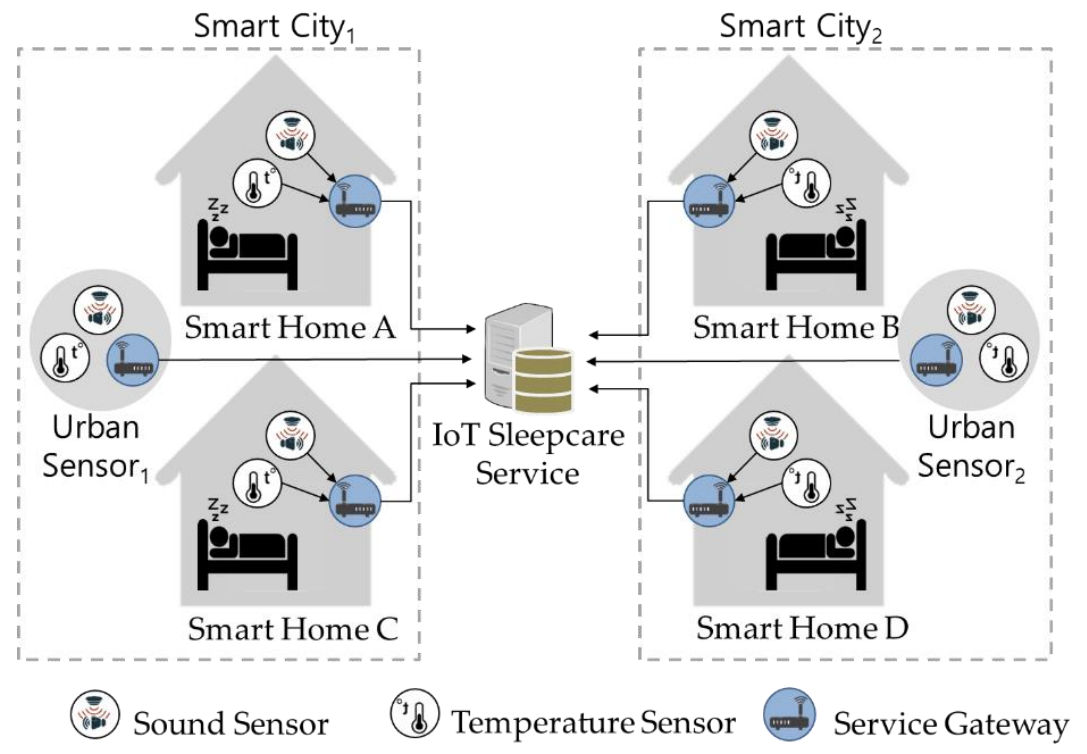

Figure 1. Example of smart home system: loT sleep care service

If smart city infrastructure can be included in the range, it can provide other services in addition to the sleep care service[12]. In this case, the smart home functions as one sensor in a smart city[13]. In addition, an urban sensor[14], which globally measures the urban environment, such as air temperature and noise, can be used. Smart Home A and Smart Home 
C are in Smart City1, and they can utilize Urban Sensor1. Similarly, Smart Homes B and Smart Home D are in Smart City2, and they can utilize Urban Sensor2[15]. As an IoT sleep care service can utilize not only the sound and temperature sensor data from each smart home, but also the temperature and environmental noise data of the entire city, it can provide area-based sleep care services.

However, the edge where sensors and services are in contact was not fully utilized. The problems mentioned in the Introduction, such as latency, overload, and service scalability, occur in the process of transmitting sensor data to a service through the edge. If a device located at the edge analyzes the data and automatically routes it, this will not only solve problems, but also enable a smart city network to be configured more efficiently. In addition, as a smart city sensor corresponds to a smart home service gateway, it can improve the quality of smart home services. Therefore, in Chapter 3, we propose and design a Smart Edge Broker that can recognize the payload of transmission traffic and actively distribute data.

\section{Smart Edge Broker}

The Smart Edge Broker proposed in this paper is an intelligent broker, and the edge located between a sensor network and service efficiently distributes traffic. It receives all the data from the sensor and analyzes the data according to the requested service and routes it to the appropriate service. Figure 4 is an example of a smart city platform when the Smart Edge Broker is introduced.

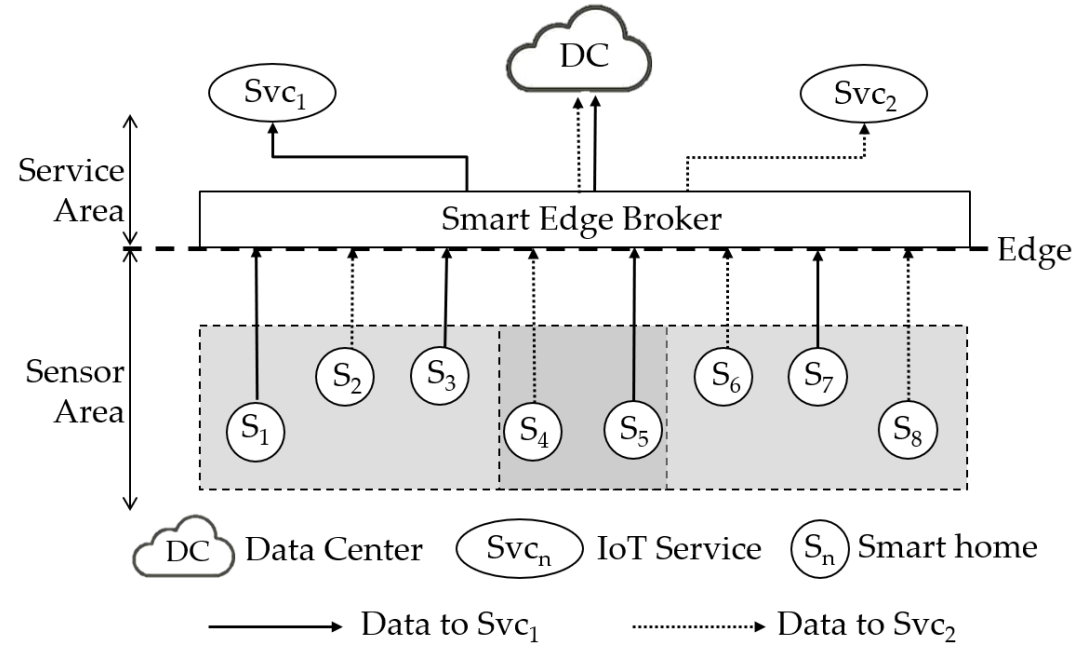

Figure 2. Smart city platform with Smart Edge Broker

The boundary points between the sensor area and service area are divided by the edge. Eight smart homes are distributed in two areas in the sensor area, and the service area consists of one data center and two services. It is assumed that Svc1 and Svc2 use the sensors installed in the smart home system to provide different services and share the sensors in the area below. It is assumed that at this time, Svc1 needs the data measured in S1, S3, S5, and S7, and Svc2 needs the data measured in S2, S4, S6, and S8. As the data center needs to collect and manage all data, it receives data from all sensors.

If this is applied to the IoT sleep care service, a smart home can correspond to a sleep care device, and a service can correspond to an IoT sleep care service. It collects the sleep data measured in each smart home and transmits it to the IoT sleep care service to provide services. 
At this time, the Smart Edge Broker provides intelligent data routing functions at the edge between the smart home and services, thereby transmitting data more efficiently.

The Smart Edge Broker has an intelligent routing function. It receives requests for desired data from each service and collects and analyzes the data from all sensors based on it. Based on the analysis results, it classifies the data requested by each service and routes it and transmits all the collected data to a data center. Figure 5 shows this process.(a) in Figure 5 shows the process by which a service receives data from a smart home and provides services.

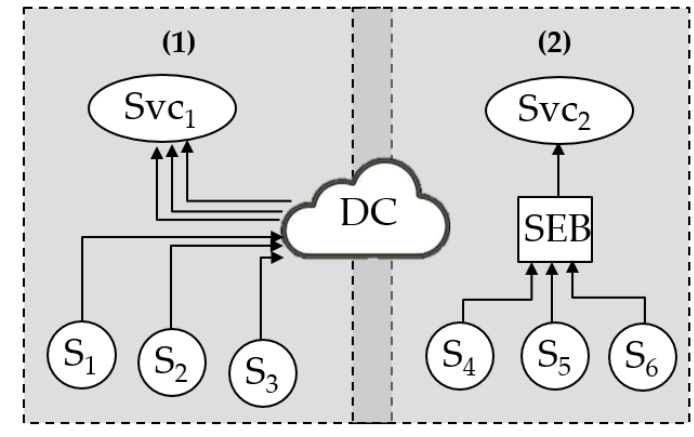

(a)

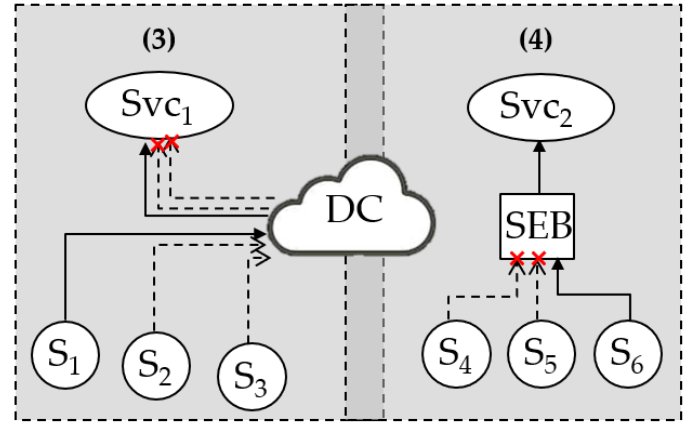

(b)

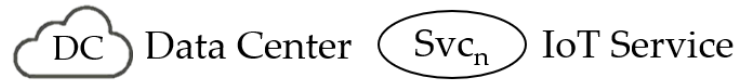

SEB Smart Edge Broker $S_{n}$ Smart Home

Figure 3. Scenarios for service routing in the Smart Edge Broker: (a) Scenario for collecting data from sensors; (b) Scenario for collecting selective data from sensors

In the case of(1), if Svc1 receives the data of S1, S2, and S3, the smart home transmits data to the data center and the service requests data from the data center and receives it. At this time, if the Smart Edge Broker brokers the data between the smart home and service, as shown in(2), there is no need to purposely transmit the data to a remote data center. Svc2 in(2) receives data of S4, S5, and S6 in the same way as Svc1 in(1), but the Smart Edge Broker does not need to go through a data center in place of the data center. In this case, the communication distance is reduced by the distance from the data center, which in turn reduces the burden on both ends of the smart home and service. In addition, as the data center does not need to collect data, there is no traffic centralization.

(b) in Figure 5 shows a case in which a service needs only some data.(3) is similar to(1), but Svc1 requests only the data of S1. In this case, Svc1 has no choice but to filter only the necessary data after receiving all the data. $\operatorname{In}(4)$, Svc2 requests the data of S6, but unlike(3), the data of S4 and S5 are filtered in advance so that the Smart Edge Broker can provide services more efficiently because there is no need to consume resources to reconstruct the data in Svc2.

\section{Conclusions}

In this paper, by proposing a Smart Edge Broker, we could solve the latency and overload problems that may occur during the process of using an IoT service through a smart home 
system and smart city platform. This is a method of analyzing and filtering the data using topic and query and routing it to the proper location.

\section{Acknowledgements}

This work was supported by the Technology development Program(Grants No. S2593303) funded by the Ministry of SMEs and Startups(MSS, Korea).

\section{References}

[1] Li, B. and $\mathrm{Yu}, \mathrm{J}$. "Research and Application on the smart home Based on Component Technologies and Internet of Things”. Procedia Engineering (2011), vol.15, pp. 2087-2092.DOI: 10.1016/j.proeng.2011.08.390

[2] Pang, Y.; Jia, S. "Wireless smart home System Based on Zigbee”. International Journal of smart home (2016), 10, pp. 209-220.

[3] Talal, M.; Zaidan, A.; Zaidan, B.; Albahri, A.; Alamoodi, A.; Albahri, O.; Alsalem, M.; Lim, C.; Tan, K.; Shir, W.; Mohammed, K. smart home-based IoT for Real-time and Secure Remote Health Monitoring of Triage and Priority System using Body sensors: Multi-driven Systematic Review. Journal of Medical Systems (2019), pp. 43. DOI:10.1007/s10916-019-1158-z

[4] Han, H.; Jo, J.; Son, Y.; Park, J. Smart sleep care system for quality sleep. (2015) International Conference on Information and Communication Technology Convergence(ICTC), Jeju Island, Korea, Oct 2015, pp. 393-398. DOI: 10.1109/ICTC.2015.7354571

[5] Skouby, K.; Lynggaard, P. smart home and smart city Solutions enabled by 5G, IoT, AAI and CoT Services. (2014) International Conference on Contemporary Computing and Informatics(IC3I), Mysore, India, Nov 2014, pp. 874-878. DOI: 10.1109/IC3I.2014.7019822

[6] Li, F.; ZHENG, B. Design of the smart city Planning System based on the Internet of Things. International Journal of smart home (2016), 10, pp. 207-218.

[7] Gaur, A.; Scotney, B.; Parr, G.; McClean, S. smart city Architecture and its Applications Based on IoT. Procedia Computer Science (2015), 52, pp. 1089-1094.DOI: 10.1016/j.procs.2015.05.122

[8] Sarkar, S. and Misra, S. Theoretical modelling of fog computing: a green computing paradigm to support IoT applications. IET Networks (2016), 5(2), pp. 23-29.DOI: 10.1049/iet-net.2015.0034

[9] Vahid Dastjerdi, A., Gupta, H., N. Calheiros, R., K. Ghosh, S. and Buyya, R. Chapter 4 - Fog Computing: principles, architectures, and applications. In Internet of Things; Morgan Kaufmann Pub: Massachusetts, United States, (2016); pp. 61-75.DOI: 10.1016/B978-0-12-805395-9.00004-6

[10] Ricquebourg, V.; Menga, D.; Durand, D.; Marhic, B.; Delahoche, L.; Logé, C. The smart home Concept : our immediate future. 2006 1ST IEEE International Conference on E-Learning in Industrial Electronics, Hammamet, Tunisia, Dec (2006), pp. 23-28.DOI: 10.1109/ICELIE.2006.347206

[11] Choi, J.H.; Kang, U.G.; Lee, B.M. Sleep Information Gathering Protocol Using CoAP for Sleep Care. Entropy (2017), 19, pp. 450.DOI: 10.3390/e19090450

[12] Lim, D.H.; Rhee, B.H. Design Study of the U-city Home Network Architecture of Cloud Computing. International Journal of smart home (2013), 7, pp. 145-156.

[13] Lee, H.J.; Kim, K.H.; Kim, Y.H. Wireless sensor Network-Based 3D Home Control System for smart home Environment. International Journal of smart home (2016), 10, pp. 159-168.

[14] Choe, Y.; Jang, M.; Kim, S. System Design for a Urban Energy Monitoring and Visualization Environment Using Ubiquitous sensor Network and Social sensor Networking. Journal of the HCI Society of Korea (2010), 5 , pp. 7.

[15] Vergura, S. "Smart City, Sustainable Mobility, Home-Work Mobility: data analysis and Actions". Renewable Energy and Power Quality Journal (2015), pp. 768-773.

[16] Catlett, C.; Beckman, P.; Sankaran, R.; Galvin, K. "Array of things: a scientific research instrument in the public way: platform design and early lessons learned". SCOPE '17 Proceedings of the 2nd International 
Workshop on Science of smart city Operations and platforms Engineering, Pittsburgh, Pennsylvania, Apr (2017), pp. 26-33.DOI: 10.1145/3063386.3063771

[17] Catlett, C.; Foster, I.; Malik, T. "Plenario: An Open Data Discovery and Exploration platform for Urban Science". Bulletin of the IEEE Computer Society Technical Committee on Data Engineering (2014), pp. 37.

[18] Cheng, B.; Solmaz, G.; Cirillo, F.; Kovacs, E.; Terasawa, K.; Kitazawa, A. FogFlow: Easy Programming of IoT Services Over Cloud and Edges for smart cities. IEEE Internet of Things Journal (2018), 5, pp. 696 - 707. DOI: 10.1109/JIOT.2017.2747214

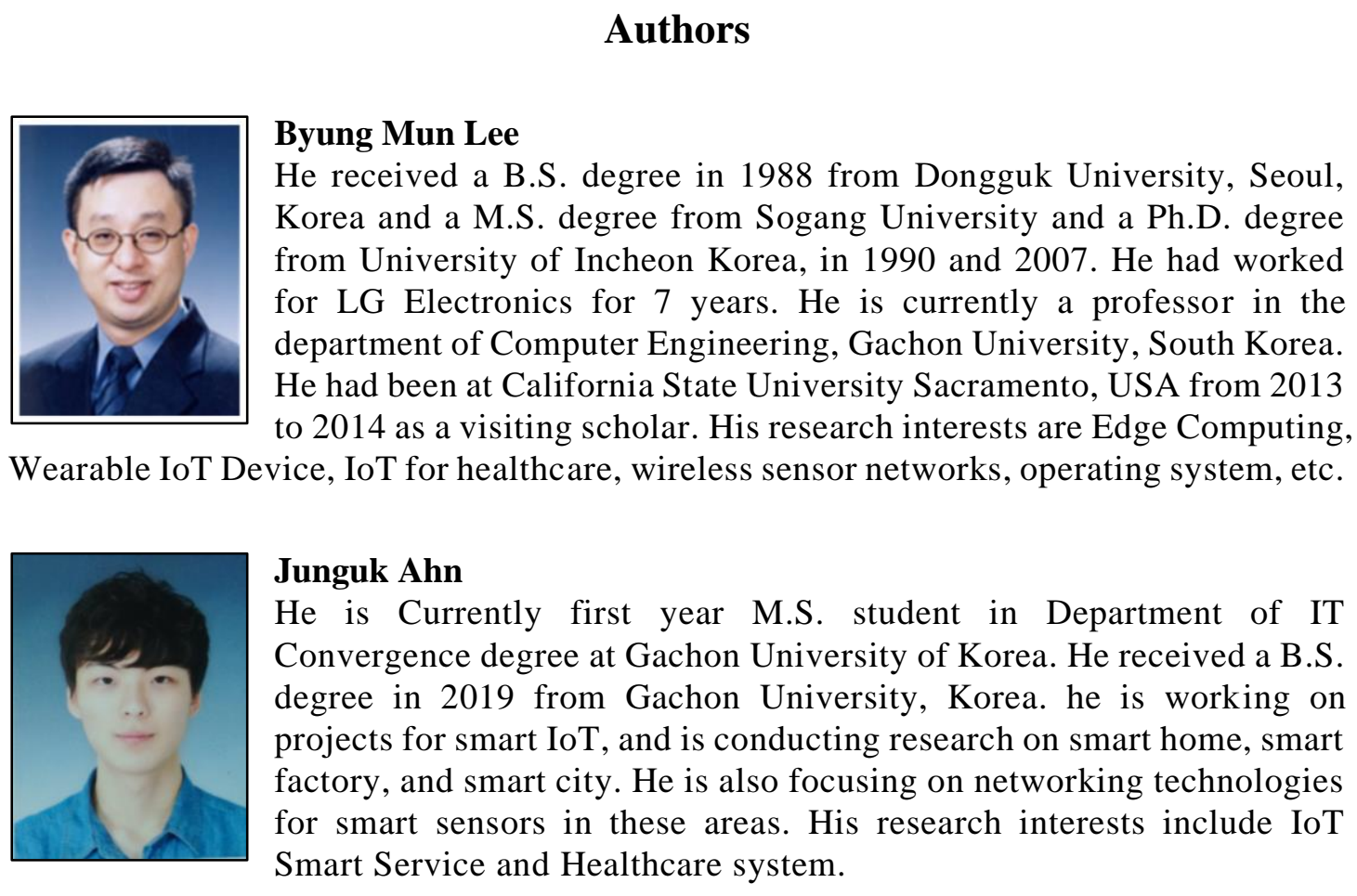

